\title{
ANALYSIS OF MECHANICAL PROPERTIES FOR THE KEY FORCE TRANSFER COMPONENTS OF 2 MN DEADWEIGHT FORCE STANDARD MACHINE
}

\author{
Wei Tieping ${ }^{1}$, Guo Jinquan ${ }^{2}$, Lai Zhengchuang ${ }^{3}$, Lin Shuo $^{4}$, Yang Xiaoxiang ${ }^{5}$ \\ ${ }^{1}$ School of Mechanical and Automotive Engineering, Fujian University of Technology, Fuzhou, China, \\ tpwei@ fjut.edu.cn \\ ${ }^{2}$ School of Mechanical Engineering and Automation, Fuzhou University, Fuzhou, China, maxguo@163.com \\ ${ }^{3}$ Fujian Province Institute of Metrology, Fuzhou, China, lzcfijl@126.com \\ ${ }^{4}$ Fujian Province Institute of Metrology, Fuzhou, China, linshuo1001@ 126.com \\ ${ }^{5}$ School of Mechanical Engineering and Automation, Fuzhou University, Fuzhou, China, yangxx@ fzu.edu.cn
}

\begin{abstract}
:
The good mechanical properties of deadweight force standard machine (DWM) are the basis to ensure the accuracy of its measurement. Aiming at the development of $2 \mathrm{MN}$ DWM, this paper carries out simulation analysis on the key components of the force value transfer process. The analysis results provide constructive suggestions for the development of $2 \mathrm{MN}$ DWM.
\end{abstract}

Keywords: Uncertainty; $2 \mathrm{MN}$ deadweight force standard machine (DWM); finite element method; mechanical properties

\section{INTRODUCTION}

With the development of economy and industrial technology, people's demand for force measurement with high precision and large range becomes more and more obvious. At present, a large number of researchers and funds have been invested in developing the force standard (base) machine as the highest standard for force traceability, and a series of static machines with different ranges $(50 \mathrm{~N}$ 4.45 MN) and measuring accuracy have been developed, including $1 \mathrm{MN}\left(U_{\text {rel }}=0.002 \%, k=2\right)$ in China, $1 \mathrm{MN}\left(U_{\text {rel }}=0.002 \%, k=2\right)$ in INRIM/Italy, $1 \mathrm{MN}\left(U_{\text {rel }}=0.002 \%, k=2\right)$ in NPL/India, $1.2 \mathrm{MN}\left(U_{\text {rel }}=0.002 \%, k=2\right)$ in NPL/UK, $2 \mathrm{MN} / 1 \mathrm{MN}\left(U_{\text {rel }}=0.002 \%, k=2\right)$ in $\mathrm{PTB} /$ Germany, $4.45 \mathrm{MN}$ in NIST/USA.

The theoretical value of uncertainty of DWM measurement is $1 \times 10^{-5}$, but the actual value is only $5 \times 10^{-5}[1]$. There are many factors affecting the measurement uncertainty of DWM, usually including the local acceleration of gravity, weight material density, uncertainty of weight mass, the density of air quality, the swing of the central boom and beam tilt (the uncertainty of DWM and the force transducer structures) [2-7]. Also, it includes the influence of tidal level effect on gravitation, the influence of air conditioning airflow, the influence of gravitation between weights, humidity, temperature, hardness of the pad, and so on [8]. Among these factors, some factors contribute more weight to the evaluation of measurement results. Some influencing factors contribute little to the evaluation of the measurement results, but they also have great influence on the measurement stability of the static crane. Among them, the strength, stiffness and stability of DWM are the most basic and the most presupposition factors.

For structure design of $2 \mathrm{MN}$ DWM in Fujian Province Institute of Metrology, its key value transfer components including the main body frame structure, the weight installation platform lifting mechanism, the structure of the inverter and the central derrick were simulated based on finite element method and the improved mechanical property solutions were put forward, which can provide constructive suggestions for the successful design of $2 \mathrm{MN}$ DWM.

\section{THE OPERATING PRINCIPLE OF 2 MN DWM}

The structure diagram of $2 \mathrm{MN}$ DWM is shown in Figure 1. The suspension system of DWM is made of an inverter and a central derrick. The gravity value of the hanging system is $40 \mathrm{kN}$. There are a total of 39 pieces of weights, which are independently added by the motors [3]. This configuration can be used to load the internal force values of $(10 \mathrm{kN} \sim 1 \mathrm{MN})$ and $(20 \mathrm{kN} \sim 2 \mathrm{MN})$ in the range increasing by the multiple of $5 \mathrm{kN}$.

Before DWM works, the inverter and the central derrick are all placed on the rack. At the same time, the weight is placed in the V-shaped block of the transmission device through the weight handle. 
While the calibration is in progress, the force transducer is placed on the moving beam to ensure that the central axis of the force transducer coincides with the central axis of the hanging system. The beam is driven upward through the roller screw by the motor until the hanging system is fully supported to reach stability by the force transducer. At this point, the hanging system is in a free state.

After that, the transfer device will transmit the required weight downward until it is completely pressed on the weight tray. Then, the force value of the weight will be transmitted to the force transducer through the hanging system. At this point, the hanging system is in the loading state.

After the verification, the servo motor reverses direction and the transfer device drives the weight to move upward until the weight is completely off the weight tray. Then, the moving beam moves downward until DWM returns to the initial state.

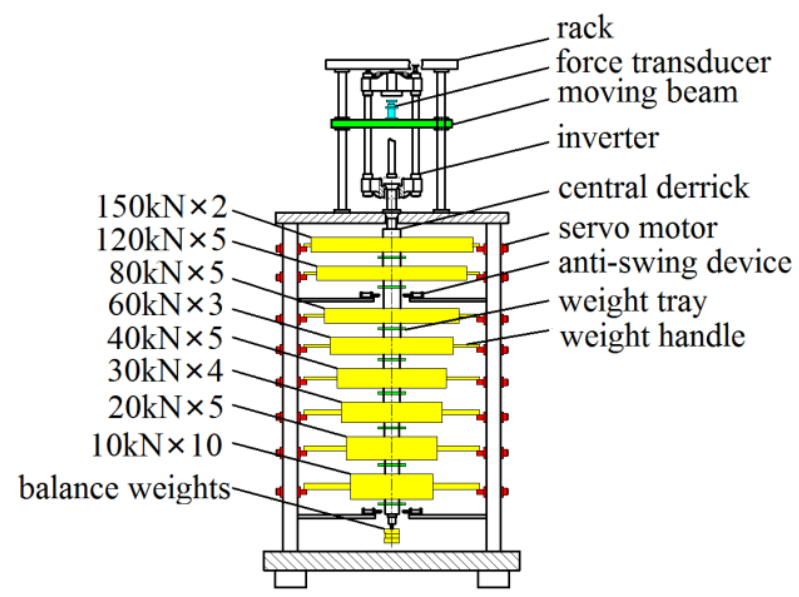

Figure 1: Structural diagram of 2 MN DWM

\section{SIMULATION ANALYSIS FOR THE KEY FORCE TRANSFER COMPONENTS}

Based on the above analysis of the working principle of 2 MN DWM, it can be known that the main frame structure, the central derrick, the inverter structure and the lifting mechanism of the weight installation platform are all the key loadbearing components. The mechanical properties of these parts directly influence the transfer effect of the value in the standard machine, which determines the measurement performance of the standard machine. The following sections will conduct finite element modelling and simulation of the key components of the design one by one. The analysis results will provide constructive suggestions for the development of $2 \mathrm{MN}$ DWM.

\subsection{The Main Frame Structures}

The main frame of the $2 \mathrm{MN}$ DWM is used for placing weights, providing static support for weights and fixing the motor that drives weights to move up and down. The main frame structure is made of the upper and lower supports, the nine columns in the middle and every three columns form a group. Five fixed bars distributed uniformly at the top and bottom are used to connect the columns to improve the stability of the structure, as shown in Figure 2. The height of the main frame reaches $10 \mathrm{~m}$, and the weight placement mechanism fixed on it has a maximum load bearing weight gravity of $2 \mathrm{MN}$. Therefore, its strength and stiffness should be analysed emphatically. In addition, the security of the connection between the weight placement mechanism and the main frame is also a key consideration. Considering the feasibility of the numerical simulation and the reliability of the analysis results, the simulation analysis can be divided into two parts: (1) the overall analysis: the strength and stiffness of the main structure are analysed, and the connection between the weight placement mechanism and the main frame is not considered; (2) the local analysis: the connection between the placement mechanism of weights and the main frame is considered, and the deformation of the main frame structure is not considered.

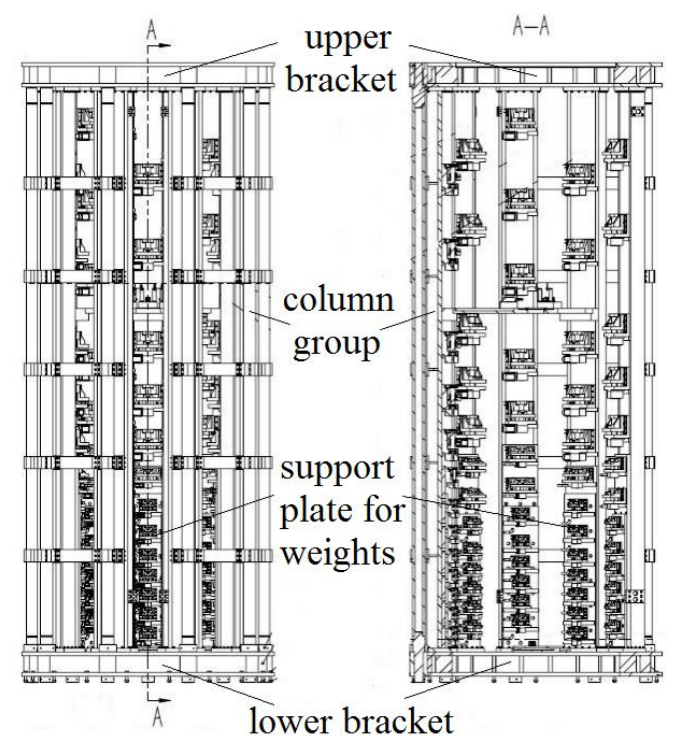

Figure 2: Schematic diagram of the main frame structure

(1) Simplification of mechanical model

As shown in Figure 3, the supporting structure for placing weights is simplified to a $\mathrm{T}$-shaped bracket for the overall analysis. It is assumed to be a rigid body, which is regarded as a whole with the main frame. The load of $2 \mathrm{MN}$ acts on the T-shaped bracket according to the distribution position of weights. As shown in Figure 4, the force value transfer mechanism of the connection between the weight placement mechanism and the main frame structure is comprehensively considered for the local analysis. On the premise of not affecting the calculation results, the deformation of the connecting column with the weight placement mechanism is ignored. Therefore, only a section of the column structure is intercepted for modelling. The connection between the belt and the column is a fixed connection. 


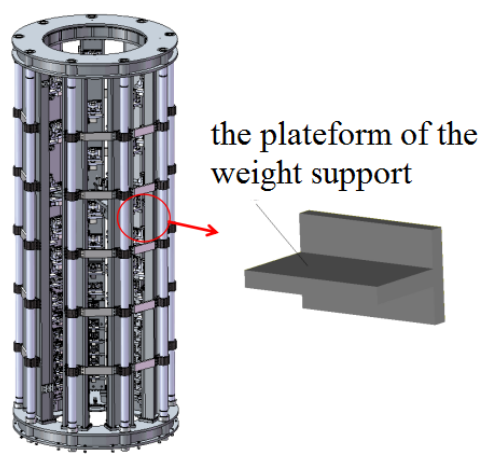

Figure 3: Simplified schematic diagram of the overall analysis structure

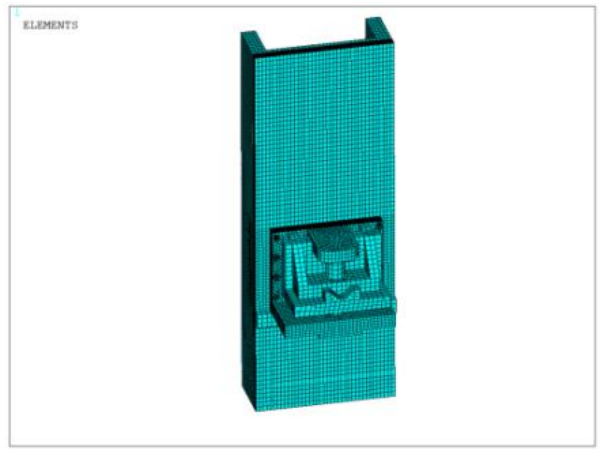

Figure 4: Simplified schematic diagram of the local analysis structure

(2) Result analysis

As shown in Figure 5, the maximum von Mises stress value of the overall analysis structure is 3.99 $\mathrm{MPa}$ at the connection position between the lower support and the column. The maximum total displacement value is $0.043 \mathrm{~mm}$ at the middle position of the column. Therefore, there are sufficient strength and stiffness for the overall frame structure to meet the measurement accuracy of DWM. As shown in Figure 6, the maximum von Mises stress value of the local analysis structure is 96.61 MPa at the edge of the hole where the uppermost screw contacts the rack, which is under the action of the maximum weight block (15-ton weight). The maximum total displacement value is $0.076 \mathrm{~mm}$ at the outer edge of the $\mathrm{V}$-shaped block.

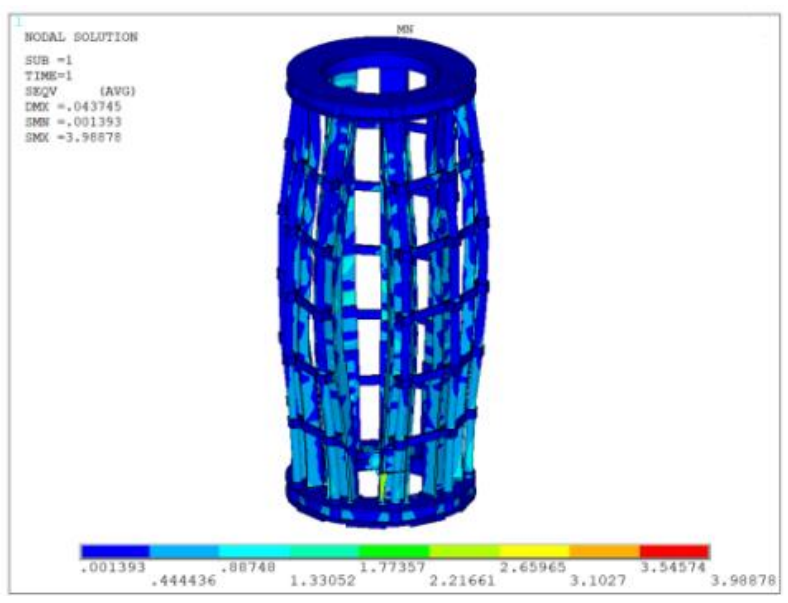

Figure 5: Stress cloud diagram of the overall frame structure

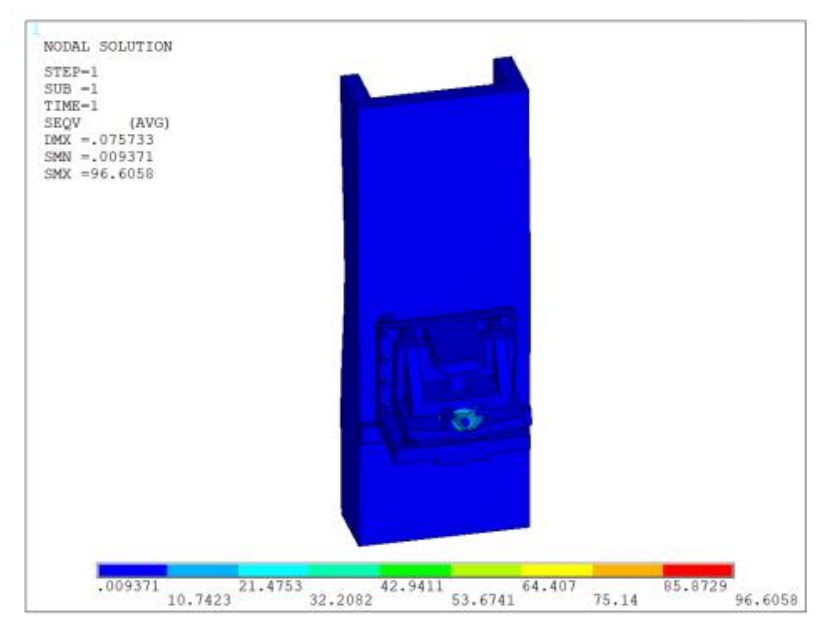

Figure 6: Stress cloud diagram of weight placement mechanism

The maximum von Mises stress value of the weight placement mechanism and its connection screws are far less than the yield limits of the corresponding materials, which indicates that the structure is able to meet sufficient strength and stiffness for the accurate measurement of DWM. In addition, the modal analysis of the first six orders of the main frame shows that the frequency of the first three orders is about $10 \mathrm{~Hz}$, and the frequency of the second three orders is about $25 \mathrm{~Hz}$, which is relatively small. Therefore, it is suggested to avoid these basic frequencies in practice to prevent resonance of the structure.

\subsection{The Central Derrick}

(1) Simplification of mechanical model

On the one hand, the structure size of the central derrick will affect the project installation. On the other hand, there are many ways to combine weights at the check points. In addition, the strength and stiffness of the central derrick directly affect the measurement performance of DWM. Therefore, it is of great importance to design the reasonable structure size for the central derrick. Here, the simulation results of the derrick with the dimensions of equal section and stepped structure are compared, which can provide theoretical basis for the structural design of the central derrick. As shown in Figure 7, the central derrick structure designed is composed of two round rods with equal sections in Figure 7 (top), while the central derrick structure designed is composed of five round rods with variable sections in Figure 7 (bottom). Both are made of 2Cr13. All levels of derrick are connected by threads and connecting rods. Each stage of derrick is installed with an equal size ring. In addition, the main function of the ring is to support the weight tray and transfer weight gravity to the hanger. 

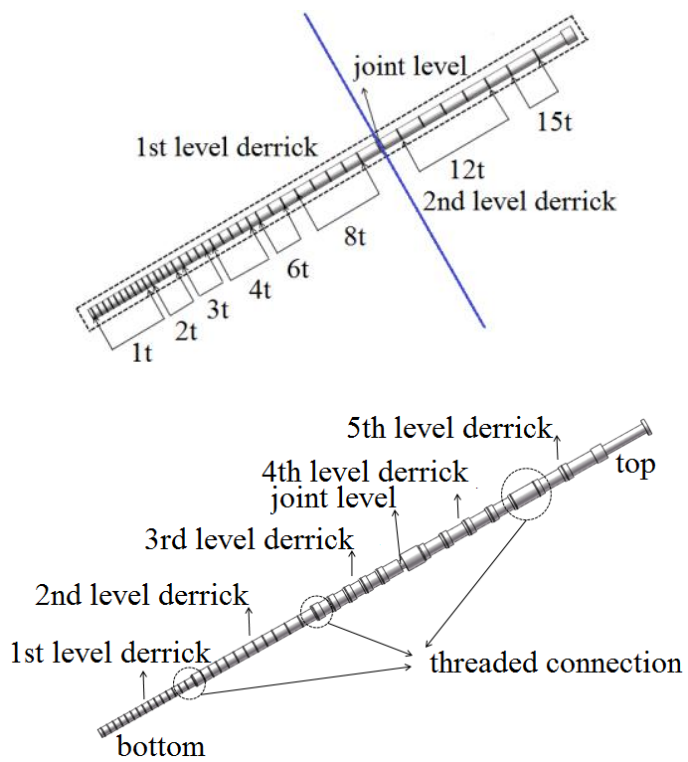

Figure 7: Structure design scheme of the central derrick, top: two round rods with equal sections, bottom: five round rods with variable sections

(2) Result analysis

The analysis results of the two design schemes are shown in Figure 8 and Figure 9. Compared with the equal-section central derrick, the von Mises stress value of the variable-section central derrick decreases by $29.99 \%$. The von Mises stress value is far smaller than the yield limit of the material and the structure obtains better strength. In addition, the total displacement value for the variable-section central derrick is reduced by $31.45 \%$, and the structure has better stiffness. Therefore, the variable-section central derrick is more in line with the actual high precision measurement requirements of DWM.

In addition, the weight should be lowered to the weight tray slowly during loading and returned to the lifting platform immediately after verification, which can reduce the creep effect of force fluctuation on the central derrick. At the same time, it is easier for the multiple levels of variable-section derrick to install. Therefore, the structural design of multi-level central derrick with variable crosssection is more in line with the actual situation.

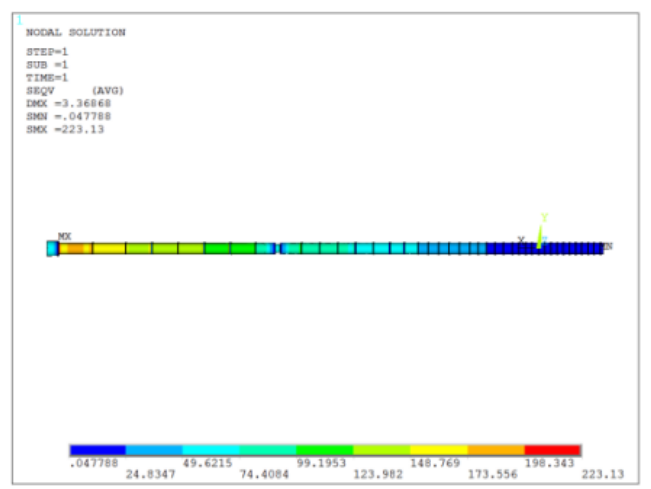

Figure 8: Stress cloud diagram of hanging structure with equal diameter central derrick

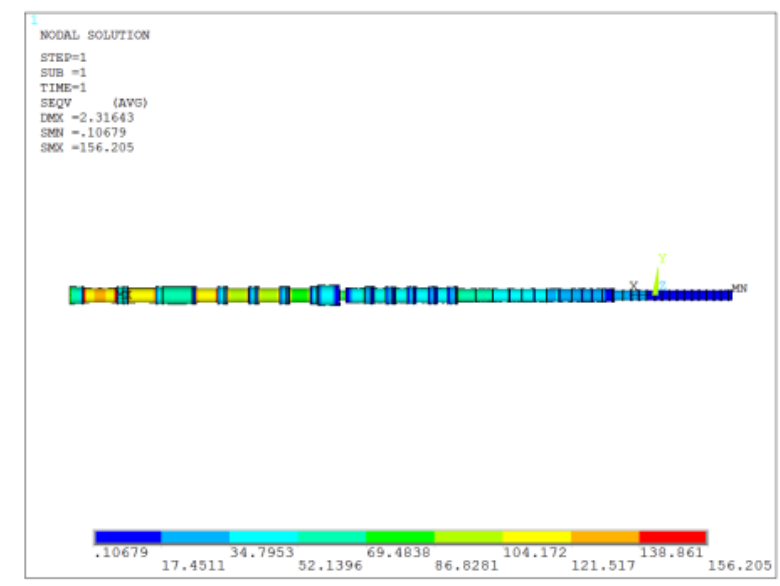

Figure 9: Stress cloud diagram of the hanging structure in the classification central derrick

\subsection{The Inverter Structure}

(1) Mechanical model

The inverter is the key load-bearing component, and its initial design structure and the structure after topology optimization are shown in Figure 10. It is very important to ensure that the structure of the inverter is subjected to uniform and stable load in the verification process. The structure consists of an upper beam, three support rods and a lower beam. Furthermore, the support rods are fixed to the top frame of the force standard device.

In the tensile state, the lower beam of the inverter can bear the force up to $2 \mathrm{MN}$, which is transmitted to the fixed platform through the support rods. Then, the tension sensor between the upper beam and the fixed platform is subjected to the weight gravity. In the pressure state, the lower beam of the inverter is subjected to the force up to $2 \mathrm{MN}$, which is transmitted to the upper beam through the support rods. Then, the compression sensor located between the upper beam and the fixed platform is begin to be verified. Here, the structure of the initial design is simulated and analysed, and the size of the initial structure is topologically optimized to obtain the optimized structure. Meanwhile, the analysis results of the two are compared.
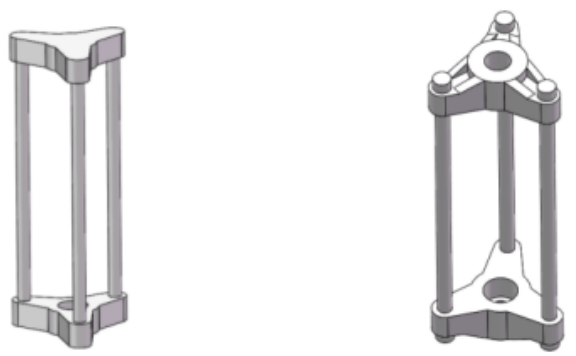

Figure 10: The inverter structure, left: initial design, right: after topological optimization

(2) Result analysis

Figure 11 and Figure 12 are the overall displacement cloud maps of the two structures before and after optimization, respectively.

It can be seen from the figures that the maximum von Mises stress value of the inverter structure at the 
contact point between the lower beam and the bearing decreases from $472 \mathrm{MPa}$ to $176 \mathrm{MPa}$, which is far less than the yield limit of the material and could meet the strength requirement. The maximum overall displacement value of the inverter structure at the contact point between the lower beam and the hanger decreases from $2.6 \mathrm{~mm}$ to $1.6 \mathrm{~mm}$. In addition, the modal analysis of the first six orders of the main frame is carried out, and it is found that the frequency of the first three orders after optimization is around $10 \mathrm{~Hz}$, which is one order larger than that the structure before optimization. While the frequencies of the second three orders before and after optimization are around $100 \mathrm{~Hz}$ with little change.

As can be seen from the above, the maximum von Mises stress value of the lower beam of the optimized inverter is obviously reduced. Furthermore, the stress distribution range of the optimized structure is smaller and the overall force is more uniform. Therefore, the optimized inverter is able to obtain a more stable working state.

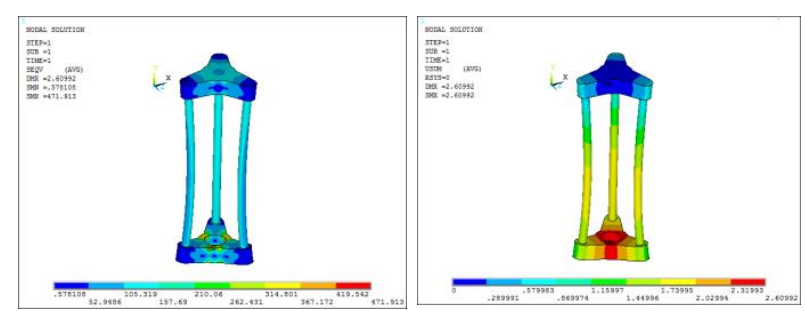

Figure 11: Analysis results of the initial design structure of the inverter, left: initial design, right: after topological optimization

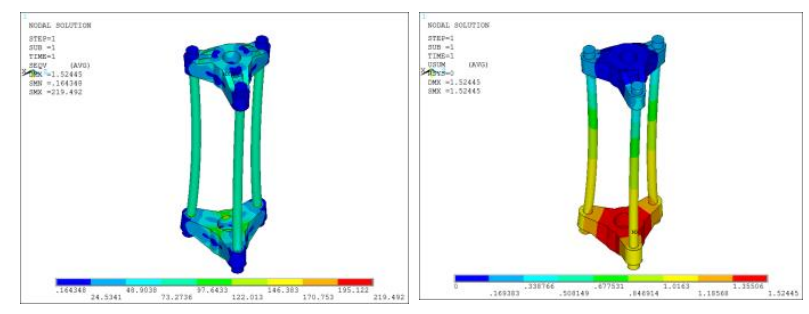

Figure 12: Analysis results of the optimization structure of the inverter, left: stress cloud diagram, right: displacement cloud diagram

\subsection{Lifting Mechanism of Weight Placing Platform}

The lifting mechanism of the weight placing platform is mainly used in the loading and unloading of DWM. Also, it is used for auxiliary lifting in the maintenance and repair, which is convenient for vertical transportation of personnel and rack appliances. As shown in Figure 13 (left), the screw rod and the screw nut constitute a screw pair, and the motor drives the screw rod to rotate and drives the lift platform connected with the screw nut to rise and fall. The maximum placement weight of $2 \mathrm{MN}$ DWM is $15 \mathrm{t}$ supported by three screw rods in a $120^{\circ}$ distribution. Considering the high lifting height of the placing platform, the slender support bar and the high safety requirement, the mechanical analysis of the structure safety must be carried out.

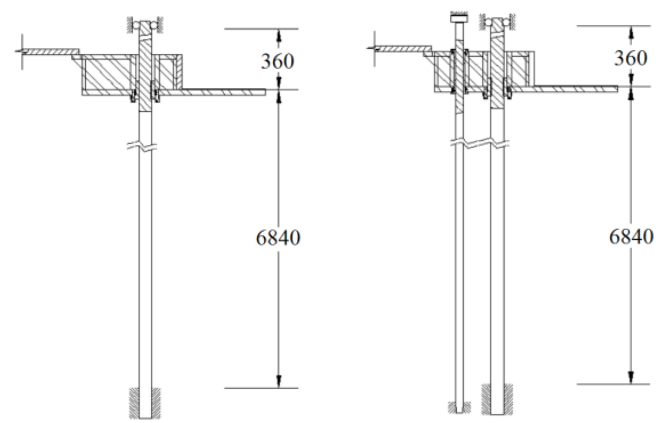

Figure 13: Schematic diagram of lifting platform mechanism for placing weight, left: without guide bars, right: with guide bars

\section{(1) Mechanical model}

The simplified model mainly considers the deformation, strength and buckling of the whole lifting mechanism of the platform, so the connection problem of the detail structure such as the strength of screw thread is not considered. Without affecting the simulation results, the platform lifting mechanism is simplified as following: 1) screw nonwork state cannot rotate, and lift platform by selflocking stays at a certain height. The top of the screw constraint torus only limits horizontal displacement, and six degrees of freedom of the bottom of the torus are all fixed ignoring the screw thread; 2) the railing of the outer ring of the lift platform is not considered, and the railing of the platform is much smaller than its own weight. Therefore, only the weight of the lifting platform itself is considered; 3 ) the disassembled plate meets the strength requirement, and it can be considered as a rigid plate for the simulation model as shown in Figure 14

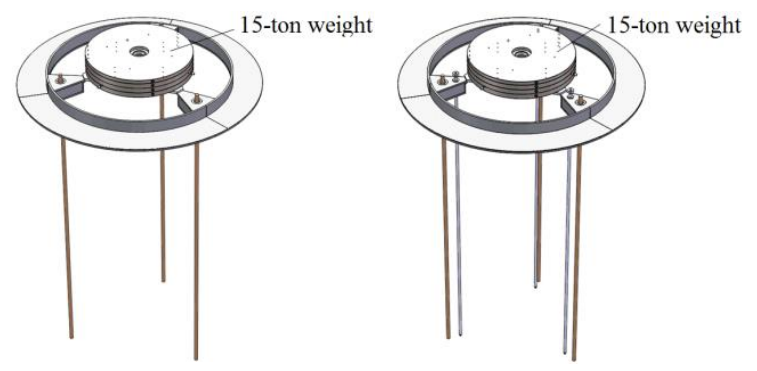

Figure 14 Simplified schematic diagram of mechanism model of lifting platform for weight, left: without guide bars, right: with guide bars

(2) Result analysis

As shown in Figure 15 (left), the maximum von Mises stress value of the mechanism without guide bars is $72.89 \mathrm{MPa}$ located at the triangular floor plate under the disassembly end supported by the platform lifting mechanism. The maximum total displacement value is $1.55 \mathrm{~mm}$ located in the middle and outer edge of the decorative pattern board. As shown in Figure 15 (right), the maximum von Mises stress value of the mechanism with guide 
bars is $66.6 \mathrm{MPa}$ at the same location as the mechanism without guide bar. The maximum total displacement value is $1.35 \mathrm{~mm}$ at the same location as the mechanism without guide bars.

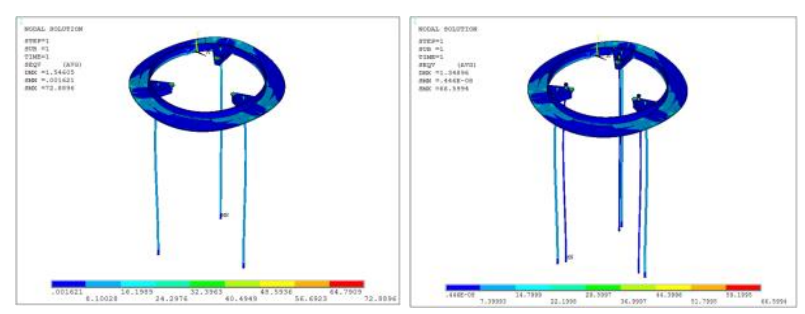

Figure 15 Finite element analysis results of platform lifting mechanism, left: without guide bars, right: with guide bars

According to the mechanical design manual, the allowable deflection of the shaft is $0.0003 l=$ $2.16 \mathrm{~mm}, l$ is the length of the bar. As we known in the Figure 15, the maximum total displacement value of the lead rod is $1.01 \mathrm{~mm}$ without guide bars and $1.11 \mathrm{~mm}$ with the guide bars. Therefore, it can be seen that the screw rods of the two kinds of structure meet the deflection requirements. At the same time, according to the Euler formula of critical load, the screw rod is determined to be a large flexibility rod. Furthermore, it is found that the first critical load is much larger than the actual maximum applied load according to the stability calculation. Therefore, the mechanism meets the stability requirement.

In addition, the lifting mechanism of the weight placing platform in the middle position is also in the extreme working condition, so the corresponding calculation and analysis should be carried out. The results show that the strength, stiffness and stability of the structure meet the requirements. Here, because of limited space of the paper, it is not elaborated.

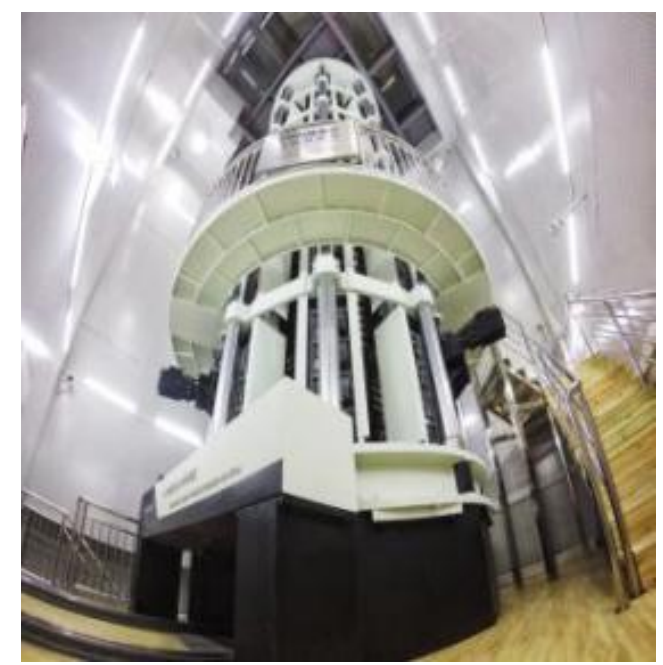

Figure 16: 2 MN DWM of Fujian Province Institute of Metrology in China

\section{2 MN DWM DEVELOPMENT RESULT}

The research results of this paper have provided a useful theoretical reference and basis for the successful development of 2 MN DWM by Fujian Province Institute of Metrology. As the largest DWM in China, its uncertainty is better than $0.002 \%$. At the same time, its performance is comparable to that of $2 \mathrm{MN}$ DWM in German PTB. Compared with the British NPL 1.2 MN DWM, its consistency is better than $0.002 \%$.

\section{CONCLUSION}

Based on the finite element method, the key structure components in the force value transmission process of $2 \mathrm{MN}$ DWM designed by Fujian Province Institute of Metrology have been simulated. The constructive suggestions are provided for its development. Furthermore, the measurement characteristics of the $2 \mathrm{MN}$ DWM will be further studied in the future.

\section{REFERENCES}

[1] D. I. Kang, B. D. Lim, "Determination of the Averaging Time for Precision Calibration of a Build-up System in a Deadweight Force Machine", Measurement, vol. 19, pp. 217-227, November 1996.

[2] A. Lindau, R. Kumme, A. Heiker, "Investigation in the Local Gravity Field of a Force Laboratory of PTB”, VDI BERICHTE, no. 1685, pp. 589-598, November 2002.

[3] R. Kumme, F. Tegtmeier, D. Röske, A. Barthel, A. Germak, P. Averlant, "Force traceability within the meganewton range", in proc. of IMEKO 22nd TC3, 15th TC5 and 3rd TC 22 International Conferences, 2014.

[4] C. Ferrero, "The measurement of parasitic components in national force standard machines", Measurement, vol. 8, no. 2, pp. 66-76, June 1990.

[5] A. Bray, G. Barbato, F. Franceschini, E. Xhomo, "Rotational and end effects: a model for uncertainty evaluation in force measurements by means of dynamometers", Measurement, vol. 17, no. 4, pp. 279-286, July 1996.

[6] Y. K. Park, D. I. Kang, J. H. Kim, M. S. Kim, "Reduction of Deadweight Pendulum Motion and its Conformation by Using a Build-up System", Journal of mechanical science and technology, vol. 23, no. 11, pp. 2956-2963, August 2009.

[7] A. Abu-Sinna, Y. K. Park, D. I. Kang, M. S. Kim, "The Influence of Loading Frame Stiffness on Loadcell-deadweight Force Machine Interaction", Measurement, vol 42, no. 6, pp. 830-835, July 2009.

[8] D. Röske, "Gravitational Forces between Weights in Force Standard Machines-Simplified Analytical and Numerical Approaches", Journal of Physics: Conference Series., vol 1065, no. 4, pp. 142012 , September 2018. 